Revue d'histoire de l'Amérique française

सES REVUE D.HISTOIRE DE L'AMÉRIQUE FRANÇAISE

\title{
Rome et l'Histoire du Canada de Brasseur de Bourbourg
}

Volume 6, numéro 1, juin 1952

URI : https://id.erudit.org/iderudit/301506ar

DOI : https://doi.org/10.7202/301506ar

Aller au sommaire du numéro

\section{Éditeur(s)}

Institut d'histoire de l'Amérique française

\section{ISSN}

0035-2357 (imprimé)

1492-1383 (numérique)

Découvrir la revue

Citer ce document

(1952). Rome et l'Histoire du Canada de Brasseur de Bourbourg. Revue d'histoire de l'Amérique française, 6(1), 112-118. https://doi.org/10.7202/301506ar d'utilisation que vous pouvez consulter en ligne.

https://apropos.erudit.org/fr/usagers/politique-dutilisation/ 
ROME ET L'Histoire du Canada DE BRASSEUR DE BOURBOURG

A) - Brasseur de Bourbourg offre un exemplaire de son oUvrage au Préfet et au Secrétaire de la Sacrée Congrégation de la Propagande

A Son Eminence le Cardinal Franzoni

Plancy (Aube), 26 juin 1852 Préfet de la S. Congrégation de la Propagande

Eminentissime Seigneur,

Avant de quitter Rome en février dernier, j'ai cherché à plusieurs reprises à présenter mes respectueux hommages à Votre Eminence, mais n'ai pas eu le bonheur de Vous rencontrer dans vos appartements. Votre Eminence voudra bien, je l'espère, apprécier les regrets que j'en éprouve, et je me croirais heureux si Elle daignait accepter, comme un sincère témoignage de mon dévouement, l'exemplaire de mon Histoire du Canada qui accompagne cette lettre. Cette histoire est écrite depuis plus de quatre ans. Si Votre Eminence daigne y jeter les yeux, Elle verra que je n'ai cessé et que je ne cesserai jamais, avec la grâce de Dieu, d'être aussi fidèle que respectueux et dévoué au Saint-Siège, à la S. Congrégation que Votre Eminence représente si Jignement, et à Votre Eminence elle-même.

C'est dans ces sentiments que j'ai l'honneur de me dire de Votre Eminence le très humble et très obéissant Serviteur et fils en Notre Seigneur. Plancy.

(Signé) L'abbé Brasseur de Bourbourg, à la Société de Saint-Victor, à

A Son Excellence Mgr Barnabd

Plancy (Aube), 26 juin 1852.

Secrétaire de la S. Congrégation de la Propagande

Monseigneur,

...Je me souviendrai toujours avec une profonde reconnaissance de l'accueil que Vous m'avez fait à Rome et du service que vous m'avez rendu en me mettant à même de partir avec la bénédiction du Souverain Pontife. Elle a commencé et elle continuera, j'espère, à me porter bonheur. Croyezle bien, Monseigneur, si j'ai commis des légèretés dans ma vie, je n'ai jamais cessé de professer entièrement les sentiments les plus catholiques et les plus dévoués au Saint-Siège et à la S.C. de la Propagande. Votre Excellence en verra la preuve dans le livre que j'ai l'honneur de Lui adresser. C'est l'Histoire du Canada que j'ai écrite, il y a quatre ans, et à laquelle j'ai mis la dernière main il y a peu de temps. A peine sortie de la presse, je me suis empressé d'en faire un paquet et de l'envoyer à Votre 
Excellence, avec un exemplaire pour Son Em. le Cardinal Préfet et un autre que je vous serais obligé de présenter Vous-même au Saint-Père avec la lettre qui y est jointe.

La Société de Saint-Victor, où je suis, est à la campagne et loin de Paris; autrement je n'aurais envoyé les livres à Rome que reliês. L'envoi aurait été fort retardé si j'avais dû les envoyer relier d'abord à Paris, et je désirais vivement Vous faire connaître ainsi qu'à Notre Très Saint-Père le genre de travaux dont je me suis presque constamment occupé. J'espère donc, Monseigneur, que mon offrande, tout humble qu'elle soit, n'en sera pas moins bien accueillie par le Pape, dont j'implore humblement la bénédiction apostolique.

Agréez, je Vous prie, Monseigneur, l'expression des sentiments respectueux avec lesquels j'ai l'honneur d'être, comme toujours, de Votre Excellence le très humble et très obêissant serviteur.

(signé) L'abbé Brasseur de Bourbourg, à la Société de Saint-Victor, à Plancy.

Archiv. S.C. Propag. Scritture riferite nei Congressi: America Settentrionale 6 (1849-1857), fol. 481 et 483.

B) - Lettre de Brasseur de Bourbourg au pape a propos de: SON OUVRAGE SUR LE CANADA.

Très Saint Père,

Prosterné humblement aux pieds de Votre Sainteté, je soussigné, prêtre, supplie Votre Béatitude de daigner agréer l'hommage d'un livre qui vient d'être terminé et dont le Pape appréciera, je l'espère, l'esprit tout catholique et dévoué au Saint Siège. Quatre mois se sont écoulés depuis que j’ai eu le bonheur d'être admis à baiser les pieds sacrés du Saint Père, et je n'ai cessé depuis lors de bénir le ciel de l'accueil paternel que j'ai reçu et des paroles pleines de bonté que Votre Sainteté a daigné m'adresser. Les sentiments que je professe dans mon Histoire du Canada, écrite depuis plus de quatre ans, sont ceux que j'ai professés toujours, surtout depuis que j'ai eu le bonheur d'entrer dans le sacerdoce. Mon obéissance absolue à l'Église et mon amour filial pour le saint et glorieux Pontife que le ciel a commis à sa tête ne failliront jamais. C'est ce que j'espère avec la grâce de Dieu et la bénédiction Apostolique de Votre Sainteté.

De Votre Béatitude, je me redis pour toujours le très-humble, trèsdévoué et très-obéissant fils en Notre Seigneur Jésus-Christ.

L'abbé Brasseur de Bourbourg, prêtre à la direction de la Société de Saint-Victor, à. Plancy.

Plancy (Aube), ce 24 juin, jour de saint Jean et saint Paul, martyres.

Archiv. S.C. Propag. Ibid., fol. $616=$ copie. 
C) - Réponse du Pape Pie IX à Brasseur de Bourbourg

PIUS PP. IX, dilecto Filio Presbytero Brasseur, Plancy (Aube). Dilecte Fili, Salutem et Apostolicam Benedictionem.

Perlatum ad Nos est exemplar operis a Te, Dilecte Fili, exarati atque in lucem publicam praesenti hoc anno cum titulo Histoire du Canada, de son Eglise et de ses Missions, Pariensibus typis editi. Et quamvis nihil de ipso adhuc degustare potuerimus, sensus tamen Tuarum litterarum, quibus opus ipsum Nobis obtulisti, sperare Nos faciunt laborem tuum piissimo quem Tibi proposuisse affirmas scopo et consilio omnino responsurum. Persolvimus idcirco Tibi pro officio gratias ac studium paternae Nostrae caritatis Apostolica confirmamus Benedictione quam omnis auspicem gratiae coelestis ipsi tibi, Dilecte Fili, amanter impertimur.

Datum Romae, apud S. Petrum, die 20 Novembris 1852, Pont. Nostri Anno VII.

Arch. S.C. Propag. Ibid., fol. $615=$ copie.

PIUS PP. IX

(Traduction française du document précédent).

PIE IX, PAPE, au cher fils BRASSEUR, prêtre, Plancy (Aube). Cher Fils, Salut et Bénédiction Apostolique.

Nous avons bien reçu un exemplaire de l'ouvrage que vous avez écrit, cher Fils, et que vous avez publié cette année aux Éditions de Paris sous le titre: Histoire du Canada, de son Eglise et de ses Missions. Bien que nous n'ayons pas encore réussi à en aborder la lecture, vos intentions exprimées dans la lettre qui accompagne cet exemplaire en hommage, nous font souhaiter que votre travail réponde tout-à-fait au but très légitime que vous nous déclarez vous y être proposé. Nous nous faisons donc un devoir de vous remercier et nous vous renouvelons l'assurance de Notre paternelle bienveillance avec la Bénédiction Apostolique que nous vous donnons avec effusion, cher Fils, comme gage de nombreuses faveurs du Ciel.

PIE IX, PAPE Donné à Rome, auprès de Saint-Pierre, le 20ème jour de novembre de l'année 1852, la septième de Notre Pontificat.

D) - Mgr C.F. Baillargeon, coadjuteur de Québec, Proteste auprès du Secrétaire de la Propagande contre l'ouvrage de Brasseur de Bourbourg et contre les louanges que celui-ci en a obTEnUes dU SAINT-SiÈge.

A Son Excellence Mgr Al. Barnabo

Québec, 3 décembre 1853. Secrétaire de la S. Congrégation de la Propagande.

Monseigneur,

Nous sommes informés que M. l'abbé Brasseur de Bourbourg s'est transportê encore une fois à Rome, qu'il y a porté une prétendue Histoire 
de l'Église du Canada, qui est son ouvrage, qu'il en a distribué un exemplaire à chacun des Cardinaux, qu'il a eu l'honneur d'en présenter une copie au Souverain Pontife; enfin qu'il a réussi à obtenir du Cardinal Asquini, et même de Sa Sainteté, des lettres laudatives! Une copie de ces deux lettres est entre nos mains.

Maintenant, Monseigneur, il est important que vous soyez averti que l'apparition de cette histoire, copiée en grande partie d'un auteur protestant, avait excité en Canada un mouvement universel de mépris et d'indignation; de mépris pour les bévues et les absurdités sans nombre qu'elle renferme; d'indignation à cause des jugemens faux, des insinuations malignes, des accusations mensongères qu'elle prodigue avec audace, en particulier contre la plupart des saints Evêques dont la mémoire est en bénédiction parmi nous, et, en général, contre le clergé de cette province. Notre affliction fut aussi grande que notre étonnement, lorsque nous apprîmes que cet ouvrage, digne tout au plus d'être classé parmi les mauvais romans historiques du jour, revêtu de la haute approbation d'un des plus illustres prélats de la France, avait trouvé son rang dans une série de livres publiés pour l'instruction et l'édification de la jeunesse, sous le beau nom de Bibliothèque Catholique.

L'archevêque de Québec crut donc qu'il était de son devoir de réclamer solennellement contre des inculpations injustes et des calomnies portant atteinte à la mémoire de ses Illustres et Vénérés prédécesseurs et à l'honneur de son clergé publiées ainsi à la face du monde catholique, avec la sanction d'un prélat de l'Église. Il s'empressa d'envoyer à l'Évêque d'Arras, dont la bonne foi avait été surprise, un long mémoire renfermant une réfutation solide et péremptoire des faussetés et des accusations calomnieuses contenues dans la prétendue Histoire de l'abbé Brasseur, conjurant ce digne Prélat, au nom de la justice et de la religion, de retirer l'approbation qu'il avait donnée à ce livre. Il adressa en même temps une copie de ce mémoire au Rédacteur en chef de L'Univers, en le priant, dans l'intérêt de la vérité, de le publier dans son journal. Le premier a gardé le silence et le second s'est contenté d'écrire une note polie à l'Archevêque de Québec. Justice a été ainsi déniée à l'innocent accusé.

Mais, si jusqu'ici le mensonge a prévalu contre nous dans la France, nous espérons que la vérité triomphera auprès du Saint-Siège; que le Sacré Collège daignera prêter l'oreille à nos justes réclamations, qu'il saura repousser la calomnie; et qu'ainsi le cœur du St Père sera consolé, en s'assurant que la mal qu'on avait publié sur ses enfans du Canada n'exista jamais au milieu d'eux.

Monseigneur l'Archevêque vous avait adressé, dans le temps, un exemplaire de son mémoire contre l'Histoire de l'abbé Brasseur. Il ne prévoyait pas alors ce qui s'est passé depuis à Rome. Maintenant, il se propose d'en envoyer copie à chacun des Cardinaux, pour détromper leurs Eminences, si toutefois elles avaient pu être induites en erreur par la lecture de l'histoire mensongère de l'Eglise du Canada. 
Nous tenons de source certaine que l'abbé Brasseur, apprenant à Rome les réclamations de l'Archevêque de Québec contre son ouvrage, a osé les attribuer à la vengeance et se poser en victime des idées gallicanes, qu'il a l'effronterie de nous attribuer. Vous êtes là, Monseigneur, pour rendre témoignage à la vérité. Vous connaissez l'évêque de Montréal [ Mgr Bourget ], l'évêque de Toronto [ $\mathrm{Mgr}$ de Charbonnel], l'évêque de Bytown [ Mgr Guigues ], l'évêque de St-Hyacinthe [Mgr Prince]. Vous me connaissez aussi, puisque j'ai eu l'honneur de représenter quelque temps, auprès du St Siège, mon digne archevêque [ Mgr Turgeon ] et tous les évêques de la Province. Vous pouvez dire si nous sommes gallicans. Non! J'ose l'attester, il n'y a pas dans toute la Province ecclésiastique de Québec un seul prêtre qui ne regarde ce que l'on appelle les libertés gallicanes comme autant d'opinions erronées, et qui ne repoussât comme une injure le nom de gallican.

Sur ce point comme sur le reste, Monseigneur, nous comptons que vous prendrez notre défense au besoin. Nous attendons ce bon service de votre amour pour la vérité, de votre générosité et de votre impartialité bien connues, et spécialement de la bienveillance que vous avez daigné nous témoigner en toutes occasions, pour laquelle aussi nous vous conservons une éternelle reconnaissance.

Agréez, Monseigneur, l'assurance du profond respect avec lequel j'ai l'honneur d'être de Votre Excellence le très-humble et très-obéissant serviteur.

(signé) † C.F. Evêque de Tloa, Coadj. de l'Archev. de Québec.

Archiv. de Propag. Ibid., fol. 608-609v.

E) - Mgr Fioramonti, Secrétaire des Lettres latines de Sa Sainteté, en réponse à une lettre du Secrétaire de la S.C. de la Propagande, lui envoie copie de la lettre de Brasseur de Bourbourg au Pape et de la réponse relative de celui-ci et lui fait remarquer que le Saint-Père n'a porté aucun jugement sur l'ouvrage en question.

Rome, 19 janvier 1854.

Non posso meglio corrispondere alle premure di V.S. Illma e Rma, che con la lettera del 17 corrente mi significa, se non che trasmettendole qui acchiuse copia della lettera che l'Abbate Brasseur scrisse li 24 Giugno 1852 a Nostro Signore, quando gli offeriva copia della sua Storia del Canadà, e copia della Lettera Pontificia in data del 20 Novembre dello stesso anno al suddetto ecclesiastico data in risposta. Da questa conoscerà che il S. Padre non ha portato alcum giudizio intorno al merito dell'opera suddetta, la quale dichiara di non aver ancor letta. Con sensi di ossequiosa e perfetta stima passo a dichiararmi di V.S. Illma e Rma... Roma 19 Gennaro 1854 (signé Domenico Fioramonti, Segr. delle Lettere Latine di N.S. Archiv. de Propag. Ibid., fol. 613. [ Le texte des deux lettres mentionnées se trouve plus haut ]. 
Mgr Fioramonti...

(Traduction)

Pour répondre à vos instances, signifiées dans votre lettre du 17 janvier, je ne puis mieux faire, Illustrissime et Révérendissime Seigneur, que de vous trasmettre, sous ce pli, copie de la lettre que l'abbé Brasseur écrivait le 24 juin 1852 à Sa Sainteté en lui offrant un exemplaire de son Histoire du Canada, et copie de la Lettre Pontificale, en date du 20 novembre de la même année, adressée en réponse au même ecclésiastique. Vous verrez dans cette dernière lettre que le Saint-Père n'a porté aucun jugement sur le mérite de l'œuvre en question, puisqu'il déclare ne pas encore l'avoir lue.

Avec des sentiments de respect et d'estime, je me déclare, Illustrissime et Révérendissime Seigneur...

Domenico Fioramonti, Sec. des Lettres Latines du S.P. Rome, le 19 janvier 1854.

F) - Répondant au Secrétaire de la Propagande le cardinal Asquini nie que sa réponse à Brassenr de Bourbourg puisse être interprétée comme une approbation de son ouvrage, qu'il n'a lu qu'en partie, et désapprouve les faussetés de celui-ci.

\section{A Mgr Barnabò}

Segr. di Propaganda

\section{Illmo e Rmo Signore,}

Mi duole moltissimo se la mia lettera responsiva al Signor Abate Brasseur de Bourborugh (sic) che mi offriva la sua opera sul Canadà siasi potuta interpretare, anziche per uno di quei complimenti, qualunque ne siano l'espressioni, che se usano in simili circostanze, per un' autorevole giudizio' sulla medesima. In essa lettera apertamente dichiarasi che l'opera non ̀̀ stata da me letta intieramente, e che solo da quel che erasi veduto potevasi dedurre bene di essa. Ecco.

D'altronde si nella mia qualifica di Cardinale, che di Prefetto di una Congrgazione estranea all'esame dea libri, e sotto qualunque altro titolo, non ho inteso mai di darvi un giudizio.

Ecco quanto ho creduto manifestare a V.S. Illma e Rma in riscontro alla lettera, che mi ha diretta sul proposito, autorizzandola anche, se Ella crede, a trasmettere questa mia in originale ai degnesissimi Prelati del Canadà dichiarando loro che io non intendo di approvare in nessun modo quanto passa essersi stato scritto nell'opera contro la verità dei fatti, $\theta$ che non sia consentaneo al rispetto loro dovuto, non che ai loro predecessori.

Dopo ciò altro non mi resta che confermarle i sentimenti della mia distinta stima con cui le baccio di cuore le mani. Di V.S. Illma e Rma... Dal Palazzo Sforza Cesarini 23 Gennaro 1854 Archiv. de Propag. Ibid., fol. 619. 
A Mgr Barnabo,

(Traduction)

Sec. de la Propagande.

Illme et Revme Seigneur,

Je déplore infiniment que ma lettre adressée à l'abbé Brasseur de Bourborugh (sic), en réponse à l'hommage qu'il me faisait de son ouvrage sur le Canada, ait pu être interprétée comme un jugement d'autorité (comme une approbation) sur cet ouvrage, et non pas comme un de ces compliments, aux formules si variées, dont on se sert en pareilles circonstances. Dans cette lettre il est ouvertement déclaré que je n'ai pas lu l'œuvre en son entier, et que, d'après les seules apparences, on pouvait penser d'elle en bien.

D'autre part, ni à titre de Cardinal, ni à titre de Préfet d'une Congrégation étrangère à l'examen des livres, ni à aucun titre, je n'ai jamais eu l'intention de porter un jugement sur le sujet.

Voilà done ce que j'ai cru bon de vous manifester, Illustrissime et Révérendissime Seigneur, en réponse à la lettre que vous m'avez adressée à ce propos. Je vous autorise aussi, si vous le croyez nécessaire, à transmettre cette lettre-ci dans sa teneur originale aux très dignes Prélats du Canada, on leur déclarant que je n'entends approuver en aucune manière tout ce qui a pu être écrit dans l'ouvrage contre la vérité des faits, et qui ne soit pas conforme au respect qui leur est dû, à eux et à leurs prédécesseurs.

Après tout ceci, il ne me reste plus qu'à vous assurer de mes sentiments d'estime particulière avec lesquels je baise cordialement vos mains.

De votre Illme et Revme

Rome, le 23 janvier 1854

(signé) F. Card. Asquini. 